up on the proximal side of the wrist-joint. A three-quarter inch incision is made extending upwards from the level of the wrist-joint directly over the ulnar border of the flexor carpi radialis tendon; this tendon is pulled to the radial side, the median nerve is gently displaced ulnarwards, and the tendon of flexor longus pollicis will be seen lying deep and between these two structures (Fig. 2). The tendon will be lying slack, and on this account may appear a little like the median nerve. The theca is incised and the tendon pulled out. Sometimes it may not slip out quite readily; this is usually due to a failure to open the theca, which is thin and not very obvious in this situation. The probe is then passed and the cut tendon drawn back into the primary wound as described above.

Fixation of the cut ends of the tendon.-Any one of the advocated methods for suturing the cut ends may be employed; it should, however, be remem. bered that adaptation of the cut ends without causing any abnormal deviations is more important than close apposition of the cut surfaces. In some cases, especially in children, it is difficult to get a good hold of the tendon with the suture. In such cases the best plan I think is to hold the cut ends of the tendon successively, at their proper level in the theca; then pass a suture from side to side of the theca piercing the tendon about a quarter of an inch from the point of section; tie the suture with sufficient firmness to hold the tendon in position. Simple iodised catgut should be used for this procedure. Though absolute apposition of the cut surfaces is not obtained by this means, the ends of the tendon lie in their normal relationship and quite close enough for satisfactory healing to take place. I have only tried this procedure once in man, and then with a good result. Some experiments I have recently made on rabbits show excellent early union after suture by this method. No further deep suture should be inserted and the skin wound is closed.

After treatment.-Position. At the completion of the operation I put the digit up flexed on a pad. This position should certainly be adopted if the fixation of the cut ends is of doubtful firmness; it also has the merit of apposing as far as possible the cut edges of the theca, which in practice cannot readily be sewn together. Further, any active movement of the flexor tendon will strain the tendon but little when the finger is in this position.

Mobilisation.-Most authorities agree in concluding that union between the divided ends of tendons under average conditions is in no sense complete until about 60 days from the time of section." After tenotomy of the tendo Achillis it is customary to wait 6 to 12 weeks before permitting full use of the limb. Taking the points into consideration, I think the best results are to be obtained if the finger is left flexed for at least a month after the operation. Massage without morement may be allowed after 10 days, but in young people if the use of the other fingers is encouraged as far as possible the general circulation of the hand will remain good. Complete mobilisation under gas should be carried out about eight weeks after the operation, and up to that time no very vigorous use of the finger should be allowed. If necessary, exercises on a pendulum machine can subsequently be given to complete the restoration of the full function of the parts.

Welbeck-street, W.

\section{A CASE OF RAT-BITE FEVER TREATED WITH NEOSALVARSAN.}

BY N. F. SURVEYOR, M.A., M.D. BOMBAY, M.R.C.P. LOND., D.P.H. CAMB.,

PROFESSOR OF BACTERIOLOGY, TRANT MEDICAI COLLEGE, HOMBAY; HONORARY PHYSICLAN, SIR JAMSFTJEE JEEJEEBHOY HOSPITAL.

RAT-BITE fever is a term applied to cases following the bite of a rat. Still, one cannot say that the term connotes an entity, simply because the number of observations on this disease are not many. However, the description of the cases published seems to favour the assumption, ret one must bear in mind the possibility that the infecting organism may, be different in some of the cases that one sees.

Recently I have had the opportunity of studying a case of this disease through the courtesy of Dr. C. Fernandes, of Bombay, under whose care the patient had come for about three months prior to my seeing her on April 11th, 1913. At this time the patient was suffering from one of the periodic attacks of the fever. She was very anæmic; the face was puffy and pasty in appearance, and on the forehead and cheeks there were reddish wheals; the ears, especially the right one, were red and œdematous; similar wheals were present on the trunk also. The temperature was $103^{\circ} \mathrm{F}$, and fever had started about two days prior to my visit.

The history was as follows. The patient, a high. class Hindoo lady, was bitten on the right ear by a rat about eight years ago (in 1906). The wound did not heal, but the ear was considerably swollen and appeared quite black in colour; ultimately it ulcerated, and the ulcer took three months to heal up completely. About a fortnight after the bite the fever commenced, and she used to have a tempera. ture varying between $99^{\circ}$ and $105^{\circ} \mathrm{F}$, and lasting for from 3 to 7 days. At first there were no eruptions noticed. However, after the ulcer on the ear had healed the fever, which was always ushered in with rigors, was accompanied by large roseolar wheals on different parts of the body, especially the face. The mucous membrane of the mouth and the fauces was also swollen and red, The attacks persisted for all these years in spite of ordinary treatment with quinine, arsenic, iron, and mercury. The patient was left weaker after each attack, and as these came on generally once or twice every month the loss of flesh and anæmia were excessive. When seen first the general appearance of the patient suggested Bright's disease, as the face was puffy and there was some odema of the extremities. Anæmia was very marked. The roseolar wheals were the most characteristic feature of the fever.

The finger blood, about 1 c.c., was twice examined by cultural tests, but no microbes could be detected. The blood was very watery and almost poured out of the puncture. No malarial parasites could be detected. The leucocyte count was: polymorphonuclears, 89 per cent.; eosinophiles, 0 per cent.; round hyalines, 1 per cent.; and lymphocytes, 10 per cent. Leucocytosis was apparently present, The liver and spleen were not enlarged. The lymphatic glands, chiefly of the neck, were enlarged during the pyrexial periods and disappeared afterwards.

On account of the periodicity of the ferer and the peculiar eruption it was thought, that perhaps the infecting organism might be allied to spirochrtæ, and some; of the patient's serum was injected into the scrotum of two rabbits with 
a riew to obtaining a culture, but with negative results. One rabbit showed a rise of temperature for a couple of days about one week after the injection. The Wassermann test was also applied with rat and guinea-pig heart antigens. The serum was inactivated before testing by Fleming's method, and the complement was supplied by the addition of a known non-syphilitic serum, which was also used as a control test. The result was a negative reaction. However, next day the serum, without inactivation, was tested according to original Fleming's method, and a positive result was obtained with both antigens. These contradictory results seem to show that perhaps the control's serum was rich in complement and the small amount of immune body in the patient's blood had not com. pletely used it up. 'That the quantity of com. plement in the serum is not a fixed amount is quite possible, because in some cases of marked syphilitic eruptions the Wassermann test as modified by Fleming has been found negative, and in such cases inactivating the serum and adding the complement from a known non-syphilitic has given a positive result. In such a case it is quite possible that the syphilitic serum must have been very rich in complement. I have actually come across such a case. The urine was found to contain albumin, a few leucocytes, granular casts, and red blood corpuscles. The last were present even during apyrexial periods, but they were much increased with the onset of fever. This seems to show that along with the roseolar state of the skin the kidneys were also similarly affected. There were always marked lumbar pains during the attacks. The menstrual flow had stopped for about two years previously.

As the patient's condition was getting worse it was decided to try an intramuscular injection of neosalvarsan. On April $16 \mathrm{th} 0.7 \mathrm{grm}$. of the drug was injected, the temperature being $99^{\circ}$. For about a week the temperature kept varying between $99^{\circ}$ and $102^{\circ}$, and there was severe pain at the site of the injection, which was moderately swollen. However, iafter that the temperature came down to normal and remained so for a fortnight; then it went up to $100^{\circ}$ only for a couple of days. There was very little roseolar eruption seen at this time. After another month a little rise occurred, but since then the fever has disappeared, and the patient has been putting on weight and getting stronger daily. The urine still contains traces of albumin, but since Sept. 7th last the red corpuscles have completely disappeared. No casts are now present.

About the time of the injection the patient's weight was $94 \mathrm{lb}$, and in September it was $118 \mathrm{lb}$., while on Nov. 11th it was $123 \mathrm{lb}$. Some two months after the injection the patient was put on metharsinate of sodium by the mouth, as she refused to have injections of any drug, and the improvement in the general condition has been steady.

Since the injection of neosalvarsan in this case I have come across the statement ${ }^{1}$ that Hata has collected eight cases of rat-bite fever treated satisfactorily with salvarsan. This seems to favour the belief that the organism is probably a protozoon, as suggested by T. J. Horder in his paper on Rat-bite Fever. ${ }^{2}$

About 12 years ago I had seen a Hindoo lad with the same fever. He, too, had roseolar eruption accompanying the temperature, and salol dissolved

1 The Medical Annual, 1913, p. 38.
2 Quarteriy Journal of Medicine, vol. 1ii. , No. 13, January, 1910. in olive oil was found to relieve itching and burning sensation. The temperature used to go up gradually to $105^{\circ}$ or $106^{\circ} \mathrm{F}$. His blood did not show any organisms at the time, and failed to give any culture. He made a complete recovery under a prolonged course of methylene blue, quinine, arsenic, and mercury, though what part the drugs played in the actual cure could not be ascertained. He has grown enormously stout now, probably due to the prolonged treatment with the drugs mentioned, especially arsenic, as there is no tendency to obesity in the family.

In conclusion, it may be stated that the rash very much resembles that in malarial fevers; however, the examination of the blood settles the point. Again, quinine rash may be easily kept under check by administration of calcium lactate, which was found of no avail in the treatment of the case of the Hindoo lady.

Bombay.

\section{atteoical Sorretics.}

\section{ROYAL SOCIETY OF MEDICINE.}

\section{SURGICAL SECTION}

Brachial Arterio-venous Aneurysm treated by Vascular Suture.-Ruptured Intestine.-Endothelioma of the Breast.

A MEETING of this section was held on Dec. 9th, Mr. G. H. Makins, the President, being in the chair.

Mr. GILBERT KeMPE read a paper on a case of Brachial Arterio-venous Aneurysm treated by Vascular Suture. It was that of a man, aged 56, who came up for examination of an injured wrist. He had an impacted fracture of the left radius in good position, but by $X$ rays the hand held six large shots. Comment on these showed that they had been received about 25 years ago. The man's wife volunteered that there were so many in the other arm (the right) "that they rattled about like a bird singing." The sound, or sensation, had been noticed for about two years Inspection showed a large pulsating swelling in the arm just below the anterior axillary fold, and in the line of the brachial artery. It was increasing in size, but was not painful. The swelling was soft and compressible, and pulsated regularly. A thrill was easily felt, which was a continuous one, but which had a systolic increase of intensity. The swelling could be traced into the axilla, and a soft pulsating swelling was found beneath the right clavicle. Here a similar thrill could be felt, but it was less marked than in the arm. Pressure on the subclavian artery above the clavicle caused a collapse of both swellings and a cessation of the thrill. Release of the pressure caused the swellings to fill up slowly, but they required several pulsations to become as full as before. Pulsation in any of the superficial veins of the arm, forearm, or thorax could not be detected. The influence of respiration on the swellings was not noted. The right radial pulse was less full than, and in time rather behind, the left. A humming, low-pitched bruit with high-pitched systolic accentuations could be heard over the swelling. The systolic notes were conducted as far as the bend of the elbow. The complete bruit could be heard, but not so loudly, beneath the right clavicle. There was no evidence of intrathoracic aneurysm. The condition presented most of the points necessary for a diagnosis of brachial arteriovenous aneurysm. It was rapidly increasing in size, even while the man was in bed and under observation. It was decided to sever the communication and to restore the ordinary state of the circulation. A dissection showed a communication between the upper part of the brachial artery and the inner of the venæ comites. The communication was not direct, for a small but distinct bulb, of the size of a cranberry, intervened between the vessels. They were therefore dealing, speaking strictly, with a varicose aneurysm. The brachial and axillary arteries above the anastomosis were of the size of a common iliac artery, while below the B B 3 